\title{
Aspectos clínicos e funcionais da Colpocefalia: um relato de caso no interior da Amazônia
}

\author{
Clinical and functional aspects of Colpocephaly: a case report in the interior of the \\ Amazonia
}

\begin{abstract}
Aspectos clínicos y funcionales de la Colpocefalia: reporte de un caso en el interior de la Amazonía
\end{abstract}

Marcella Né Pedrosa de Magalhães ${ }^{1 *}$, Keliane Serra Portela ${ }^{1}$, Paulo Henrique Ramos Pimentel ${ }^{1}$, Innocencio do Nascimento e Silva Neto ${ }^{1}$, Suelen Andrade Ricarte ${ }^{1}$.

\section{RESUMO}

Objetivo: Relatar as características clínicas e funcionais de uma criança portadora de Colpocefalia no interior da Amazônia. Detalhamento do caso: Criança, do sexo masculino, idade de 2 anos e 8 meses, com acompanhamento multiprofissional, apresentou o diagnóstico de Colpocefalia associada à microcefalia. Nascido à termo, de 41 semanas por parto via vaginal, com baixo peso e circunferência cefálica de $33 \mathrm{~cm}$, sendo internado por infecção neonatal durante 14 dias, atualmente ao exame físico apresenta-se normotônico, sem bloqueios articulares, com perímetro cefálico de $45 \mathrm{~cm}$, hiperreflexia patelar e aquileu, presença de Sinal de Babinski, pé em inversão e plano, não engatinha, não fica em pé sozinho e não deambula. Avaliou-se também o desenvolvimento neuropsicomotor, através da Escala Baylely, a criança obteve as seguintes pontuações escalonadas de acordo com os cinco domínios: cognição (1), linguagem (8), motricidade (4), socioemocional (6) e comportamento adaptativo (22). A presente escala teve o potencial de fornecer informações clinicamente úteis sobre a doença em questão. Considerações finais: Detectou-se através da avaliação atraso do desenvolvimento neuropsicomotor, entretanto, não é possível afirmar que este seja decorrente da Colpocefalia, pois a criança apresenta múltiplas comorbidades que podem agravar as taxas de atraso no desenvolvimento neuropsicomotor.

Palavras-Chave: Sistema nervoso central, Anormalidades congênitas, Deficiências do desenvolvimento, Exame físico.

\section{ABSTRACT}

Objective: To report the clinical and functional characteristics of a child with Colpocephaly in the interior of the Amazonia. Case report: Child, male, aged 2 years and 8 months, with multiprofessional follow-up, presented the diagnosis of Colpocephaly associated with microcephaly. A 41-week-old atherm born by vaginal delivery, with a low birth weight and a head circumference of 33, being hospitalized for neonatal infection for 14 days. Currently the physical examination is normotonic, without joint blocks, with a $45 \mathrm{~cm}$ cephalic perimeter, hyperreflexia. patellar and Achilles, presence of Babinski's Sign, foot in inversion and flat, not crawling, not standing alone and not walking. The neuropsychomotor development was also evaluated through the Baylely Scale. The child obtained the following staggered scores according to the five domains: cognition (1), language (8), motor skills (4), socioemotional (6) and adaptive behavior (22), this Scale has the potential to provide clinically useful information on the disease in question. Final considerations: It was detected through the evaluation delayed neuropsychomotor development, however, it is not possible to state that this is due to Colpocephaly, because the child has multiple comorbidities that can aggravate the neuropsychomotor development delay rates.

Key Words: Central nervous system, Congenital abnormalities, Developmental disabilities, Physical examination.

1 Universidade do Estado do Pará (UEPA), Santarém- PA. *E-mail: marcela-ne@hotmail.com

SUBMETIDO EM: 10/2019 | ACEITO EM: 11/2019 ｜ PUBLICADO EM: 1/2020 


\section{RESUMEN}

Objetivo: Informar las características clínicas y funcionales de un niño con Colpocefalia en el interior de la Amazonía. Detalles del caso: Niño, hombre, de 2 años y 8 meses, con seguimiento multiprofesional, presentó el diagnóstico de Colpocefalia asociada con microcefalia. Nació con 41 semanas por parto vaginal, con bajo peso al nacer y circunferencia de la cabeza de $33 \mathrm{~cm}$, hospitalizado por infección neonatal durante 14 días. Actualmente el examen físico es normotónico, sin bloqueo articular, con perímetro cefálico de $45 \mathrm{~cm}$, hiperreflexia patelar y Aquiles, presencia del signo de Babinski, pies invertidos y plano, sin gatear, sin estar solo y sin caminar. El desarrollo neuropsicomotor también se evaluó mediante la Escala de Baylely. El niño obtuvo las siguientes puntuaciones escalonadas de acuerdo con los cinco dominios: cognición (1), lenguaje (8), habilidades motoras (4), socioemocional (6) y comportamiento adaptativo (22), esta Escala tenía el potencial de proporcionar información clínicamente útil sobre la enfermedad en cuestión. Consideraciones finales: se detectó a través de la evaluación del desarrollo neuropsicomotor retardado, sin embargo, no es posible afirmar que esto se debe a la Colpocefalia, porque el niño tiene múltiples comorbilidades que pueden agravar las tasas de retraso del desarrollo neuropsicomotor.

Palabras Clave: Sistema nervioso central, Anomalias congénitas, Discapacidade del desarrollo, Examen físico.

\section{INTRODUÇÃO}

A Colpocefalia é uma doença rara que afeta o Sistema Nervoso Central (SNC) (SALDANHA RP, et al., 2017). O termo é utilizado para descrever uma desordem neurológica na qual se tem um aumento anormal dos cornos occipitais dos ventrículos laterais. Essa anormalidade do SNC foi descrita pela primeira vez em 1940 por Benda, que caracterizou essa anomalia como uma vesiculopatia, mas foi em 1946 que Yakovlev e Wadsworth inseriram o termo Colpocefalia, a partir do grego "Kolpos", oco (PUVABANDITSIN S, et al., 2006).

Essa desordem é uma doença congênita que se caracteriza por um raro achado anatômico no cérebro, que quase sempre se manifesta bilateralmente, porém com possibilidade de se apresentar unilateralmente (SARNAT HB, 2002). É uma doença occipital seletiva, não progressiva e não obstrutiva, não necessitando assim de correção cirúrgica (ULUDAG S, et al., 2012).

Acredita-se que essa má formação congênita aconteça em virtude de um distúrbio na proliferação, migração neuronal na embriogênese ou organização do SNC (PATNAIK A, et al., 2002). Sua fisiopatologia é associada ao subdesenvolvimento ou a falta de espessamento do tecido nervoso no período embrionário que ocorre entre o segundo e o quinto mês de vida intrauterina (ULUDAG S, et al., 2012).

Existem múltiplas etiologias que podem estar relacionadas com esse desenvolvimento anormal do SNC, dentre elas estão: injúrias intrauterinas como infecções por vírus (toxoplasmose, citomegalovírus, zika vírus), perinatais como hipóxia neonatal, uso de medicamentos (corticosteroides, salbutamol e teofilina) pela mãe durante a gravidez e desordens genéticas como mosaicismo trissomia-8 e mosaicismo trissomia-9, além de erros na morfogênese (PUVABANDITSIN S, et al., 2006; PETRIBU NCL, et al., 2018).

Essa doença foi encontrada em associação com outras más formações neurológicas, sendo a agenesia do corpo caloso uma das mais frequentes, podendo ainda aparecer relacionada a distúrbios da migração neuronal, esquizencefalia, microgiria, macrogiria, aumento da cisterna magna, atrofia cerebelar, hipoplasia do nervo óptico, coloboma coriorretiniana, microcefalia, meningomielocele e hidrocefalia. (ULUDAG S, et al., 2012; PUVABANDITSIN S, et al., 2006).

Dessa forma, o quadro clínico pode ser muito variável, dependendo se tem ou não alguma outra patologia associada (ULUDAG S, et al., 2012). Geralmente, a criança portadora de Colpocefalia pode apresentar atraso no desenvolvimento neuropsicomotor, diferentes graus de deficiência mental, convulsões, alterações visuais, além de déficits motores (PUVABANDITSIN S, et al., 2006).

Dentre os instrumentos disponíveis para avaliar o desenvolvimento neuropsicomotor tem-se as Escalas Bayley de Desenvolvimento Infantil (Bayley I, II, III), sendo a versão Bayley III a mais atual, tendo surgida através da revisão da escala Bayley II (RODRIGUES OMPR, 2012). Essa revisão foi feita para melhorar a 
qualidade e enriquecer a utilidade do instrumento, os objetivos da revisão foram atualizar os dados normativos; desenvolver cinco escalas distintas: cognitiva, linguagem- subdividida em comunicação receptiva (CR) e comunicação expressiva (CE), motora- subdividida em motricidade grossa (MG) e motricidade fina (MF), sociemocional e comportamento adaptativo; reforçar a qualidade psicométrica; melhorar a utilidade clínica; simplicar os procedimentos de administração; atualizar a administração de itens e os matérias de estímulos; além de manter as qualidade básicas das Escalas Bayley (BAYLEY N, 2018).

Assim, o presente artigo tem como objetivo relatar as características clínicas e funcionais de uma criança portadora de Colpocefalia no interior da Amazônia.

\section{DETALHAMENTO DO CASO}

Criança, do sexo masculino, com idade de 2 anos e 8 meses, procedente da cidade de Santarém, no estado do Pará, com acompanhamento multiprofissional pela Associação de Pais e Amigos dos Excepcionais (APAE- Santarém), apresentou o diagnóstico clínico de Colpocefalia associada à microcefalia. Genitora, na terceira gestação, tendo dois partos e nenhum aborto, com idade de 40 anos ao engravidar, descobriu a gravidez na $24^{a}$ semana, a mesma realizou três consultas de pré-natal, não fazendo uso de ácido fólico e sulfato ferroso, teve sorologias negativas para sífilis, vírus da imunodeficiência humana (HIV), hepatite B e toxoplasmose e relata doença febril aguda de curta duração aos sete meses de gestação. Nascido a termo, de 41 semanas por parto vaginal, com baixo peso e circunferência cefálica de $33 \mathrm{~cm}$, sendo internado por infecção neonatal por 14 dias. Mãe começou a notar anormalidade por volta dos 7 meses de vida, pois a criança não apresentava controle cefálico, período em que foi encaminhada para o neurologista que atestou no dia 05/01/2018 o diagnóstico de microcefalia e Colpocefalia, sendo a última evidenciada pela tomografia computadorizada de crânio (Figura 1), além de diagnóstico de paralisia cerebral e transtorno global do desenvolvimento (TGD).

Figura 1 - Tomografia computadorizada do crânio da criança aos 7 meses de idade.

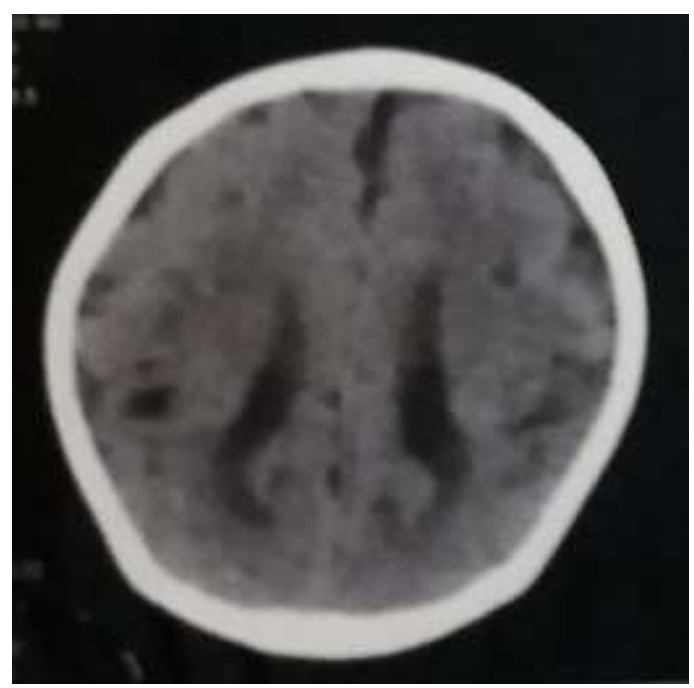

Fonte: Magalhães MNP, et al., 2019.

Atualmente ao exame físico a criança apresenta-se: com paraparesia não espástica, normotônica, sem bloqueios articulares, com perímetro cefálico de $45 \mathrm{~cm}$ (escore $Z$ de -3 ), hiperreflexia bilateral patelar e aquileu, presença de Sinal de Babinski bilateral, pés valgos e planos. Quanto ao desenvolvimento motor: não engatinha, não fica em pé sem apoio e não deambula, caracterizando atraso no desenvolvimento neuropsicomotor esperado para a idade. $E$ ao exame oftalmológico realizado por uma médica oftalmologista pediátrica a criança apresentou astigmatismo bilateral. A criança recebe atendimentos de fisioterapia, fonoaudiologia e psicologia na APAE desde os 7 meses de idade, com frequência de duas vezes por semana. 
Durante a avaliação foi aplicada a escala de desenvolvimento infantil Bayley III, em que a criança obteve as pontuações apresentadas na Tabela 1.

Tabela 1- Resultados obtidos na avaliação da Escala Bayley (3를 edição).

\begin{tabular}{|c|c|c|c|c|c|}
\hline & $\begin{array}{l}\text { Pontuação } \\
\text { bruta total }\end{array}$ & $\begin{array}{l}\text { Pontuação } \\
\text { escalonada }\end{array}$ & $\begin{array}{l}\text { Pontuação } \\
\text { composta }\end{array}$ & $\begin{array}{l}\text { Classificação } \\
\text { em percentil }\end{array}$ & $\begin{array}{c}\text { Intervalo de } \\
\text { confiança de } \\
95 \%\end{array}$ \\
\hline Cognitivo & 33 & 1 & 55 & 0,1 & $51-67$ \\
\hline $\begin{array}{l}\text { Linguagem (CR } \\
+C E)\end{array}$ & $\begin{array}{l}\text { CR: } 15 \\
\text { CE: } 22\end{array}$ & $\begin{array}{l}\text { CR: } 3 \\
\text { CE: } 5\end{array}$ & 65 & 1 & $60-75$ \\
\hline $\begin{array}{c}\text { Motora } \\
(M F+M G)\end{array}$ & $\begin{array}{l}\text { MF: } 25 \\
\text { MG: } 28\end{array}$ & $\begin{array}{l}\text { MF: } 2 \\
\text { MG: } 2\end{array}$ & 52 & 0,1 & $48-63$ \\
\hline Socioemocional & 117 & 6 & 80 & 9 & $74-90$ \\
\hline $\begin{array}{l}\text { Comportamento } \\
\text { adaptativo }\end{array}$ & 188 & 22 & 48 & $>0,1$ & $43-53$ \\
\hline
\end{tabular}

Fonte: Magalhães MNP, et al., 2019

Pesquisa submetida e aprovada quanto aos procedimentos éticos pelo Comitê de Ética em Pesquisa da Universidade do Estado do Pará, Campus Santarém, com o CAAE: 13762519.3.0000.5168.

\section{DISCUSSÃO}

A Colpocefalia é uma doença com várias possíveis causas relacionadas: congênitas, genéticas, erros de morfogênese e uso de medicamentos pela mãe (PUVABANDITSIN S, et al., 2006). Não se conhece a etiologia exata da doença na criança supracitada. Contudo, a idade tardia da genitora ao engravidar (40 anos) pode ter relação com a malformação congênita, como visto por Melo WA (2010) em sua pesquisa, que mães tardias possuem mais chances de gerar filhos com anomalias congênitas, quando comparadas a mães com idade entre 20 a 34 anos.

Essa desordem neurológica pode estar associada com outras malformações como a microcefalia, encontrada no estudo, e o mesmo foi identificado por Patnaik A, et al. (2012) que também o descreveu em seu estudo. Petribu NCL (2018) encontrou uma frequência de $62,5 \%$ da associação entre microcefalia e Colpocefalia em sua amostra $(n=10)$. Outra característica importante encontrada foi essa doença aparecer bilateralmente no cérebro, como Uludag S, et al. (2012) afirmaram ser a forma mais vista da patologia.

Quanto aos aspectos clínicos não foi encontrado na literatura informações quanto ao grau de fraqueza muscular, nem quanto ao grau de reflexos profundos para esse público. Enquanto ao tônus muscular Puvabanditsin S, et al. (2006) relataram em seu estudo de caso a presença de tônus muscular anormal da parte inferior das extremidades, fato que se diferencia do estudo em questão, no qual a criança se apresentou normotônica, assim como visto por Cerullo A, et al. (2000) em uma das crianças do seu estudo. Já quanto ao Sinal de Babinski bilateral apresentado pelo participante da pesquisa, esse dado foi compatível com o encontrado por Cerullo A, et al. (2000) exceto pelo fato de ser bilateral.

Em relação ao desenvolvimento neuropsicomotor alguns autores discorrem sobre o assunto, como Saldanha RP (2017) que relata o desenvolvimento normal para a idade da criança do seu estudo, assim como no caso relatado por Patnaik A, et al. (2012) em que a criança aos 9 meses de idade acompanhava o desenvolvimento esperado para a idade, fatos que são opostos aos encontrados na presente pesquisa, na qual o participante possui atraso persistente do desenvolvimento neuropsicomotor, devido possivelmente as suas múltiplas deficiências apresentadas.

O desenvolvimento neuropsicomotor do participante dessa pesquisa foi avaliado por meio das Escalas Bayley de Desenvolvimento Infantil (Bayley III), e de acordo com Jackson BT, et al. (2012) essa escala está entre os melhores instrumentos de avaliação do desenvolvimento infantil. Pois, ela avalia a criança dentro de 
uma escala ampliada, de 16 dias a 42 meses e 15 dias de vida, em cinco domínios: o cognitivo, o da linguagem, o motor, o socioemocional e o comportamento adaptativo. Essa escala fornece uma pontuação total bruta para cada domínio avaliado, entretanto cada domínio possui uma quantidade desigual de itens a serem avaliados, dificultando a comparação precisa entre os domínios, assim, as comparações entre as pontuações de uma criança e as de seus pares são baseadas da melhor maneira em pontuações derivadas e padronizadas: pontuações escalonadas, pontuações compostas, classificação em percentil equivalente de idade de desenvolvimento.

As pontuações escalonadas representam o desempenho, em cada subteste separado, de uma criança em relação a crianças da mesma faixa etária. Enquanto as pontuações compostas são baseadas em várias somas de pontuações escalonadas para os substestes de linguagem, motricidade e comportamento adaptativo, por outro lado para os eixos cognitivo e sociemocional é realizado equivalentes de pontuações escalonadas para pontuações compostas. E a classificação em percentil indica a porcentagem de indivíduos na amostra padronizada em uma determinada idade, da escala Bayley III, que obtiveram pontuações menores ou iguais a uma determinada pontuação escalonada ou pontuação composta (BAYLEY N, 2018).

A pontuação escalonada é expressa por um escore que varia de 1 a 19 pontos, com média de 10 e desvio padrão de \pm 3 , assim, valores entre 4 a 7 pontos são classificados como comprometimento leve, entre 3 a 1 ponto como comprometimento moderado e sem pontuação como comprometimento grave (COPPEDE AC, et al., 2012). Já a pontuação composta varia de 40 a 160 pontos, com média de referência de 100 e desvio padrão de \pm 15 . A escala preconiza que o desempenho seja classificado como muito superior para valores igual ou maior que 130, superior para valores entre 120-129, médio-alto para valores entre 110-119, médio para valores entre 90-109, médio-baixo para valores entre 80-89, limítrofe para valores entre 70-79 e extremamente baixo para valores igual ou menor que 69 (BAYLEY N, 2018).

Analisando os resultados obtidos pela criança na Bayley III, pode-se inferir que ela possui comprometimento moderado para os subtestes de cognição, CR, MF e MG e comprometimento leve para o subteste de CE e sociemocional, já em relação a crianças de idade comparável, ela obteve uma pontuação escalonada inferior ao esperado em todos os domínios (cognitivo, linguagem, motor, sociemocional e comportamento adaptativo) avaliados. No que tange a pontuação composta, obteve escore considerado extremamente baixo para o eixo de cognição, motricidade e comportamento adaptativo, escore limítrofe para o eixo de linguagem e escore na média para o eixo sociemocional, todos calculados levando em consideração o intervalo de confiança de $95 \%$. Entretanto, esses resultados foram obtidos quando comparados a crianças sem atraso do desenvolvimento, o que segundo o manual técnico da escala Bayley III, era o esperado pra uma criança com TGD.

Em um estudo realizado para a validação da escala, visto no manual técnico da terceira edição Bayley $\mathrm{N}$ (2018) com 70 crianças com idades entre 16 meses a 42 meses, com diagnóstico de TGD, avaliados pela escala Bayley III, observou-se que os domínios com menores médias foram o sociemocional e o de comunicação receptiva e expressiva, o que diferiu do presente estudo, pois o domínio com menor valor na criança avaliada foi o domínio da motricidade. Isso pode se dever ao fato de que na pesquisa realizada pela Bayley, não foram incluídas crianças portadoras de paralisia cerebral, que é um dos diagnósticos obtidos pelo participante dessa pesquisa, além de possuir outros diagnósticos correlatos ao TGD.

Em outra pesquisa feita por Bayley N (2018) com 73 crianças com idade variando entre 5 meses a 42 meses, com diagnóstico de paralisia cerebral, excluindo-se crianças com TGD, avaliadas pela escala Bayley III, viu-se que as crianças com esse diagnóstico quando comparadas ao grupo controle obtiveram pontuações significativamente menores em todos as pontuações escalonadas, assim como visto na presente pesquisa. Outro importante achado na pesquisa de Bayley foi que a pontuação composta foi menor no eixo da motricidade do que da linguagem, o que também corrobora com o encontrado no presente relato. Entretanto, quando comparado habilidades de motricidade fina e motricidade grossa, observou-se no estudo da Bayley, que o padrão motor axial foi mais comprometido que o motor apendicular em crianças com paralisia cerebral, fato que difere do encontrado no estudo em questão, onde foi visto graus de comprometimento iguais entre motricidade fina e motricidade grossa para a criança em estudo. 
Entre as limitações encontradas no estudo tem-se a pouca literatura disponível sobre a avaliação do desenvolvimento neuropsicomotor da criança com a doença de Colpocefalia, além de não se encontrar em artigos a escala Bayley III sendo utilizada em crianças com paralisia cerebral ou transtorno global do desenvolvimento. $\mathrm{O}$ que justifica o uso de literaturas não atualizadas para discorrer sobre 0 assunto no presente artigo.

Encontrou-se na literatura estudos sobre correlação da escala Bayley III com a escala Brunet- Lézine (CARDOSO FGC, et al., 2017), pesquisas utilizando a escala Bayley $3^{\text {a }}$ edição para avaliar e comparar o desempenho motor e cognitivo entre crianças de creches privadas e públicas (SANTOS MM, et al., 2013), essa escala também foi usada para comparar o desempenho motor fino entre crianças com Síndrome de Donw e crianças típicas (COPPEDE AC, et al., 2012), foi utilizado esse instrumento em estudos para avaliar o desempenho motor de lactentes frequentadores de creches (BALTIERI L, et al., 2010; SOUZA CT, et al., 2010), outra pesquisa utilizando a presente escala foi para avaliar a prevalência de atraso em prematuros de muito baixo peso (FERNANDES LV, et al., 2012).

Detectou-se, portanto, através da avaliação clínica e funcional, um atraso do desenvolvimento neuropsicomotor, entretanto, não é possível afirmar que este seja decorrente apenas da Colpocefalia, pois a criança apresenta múltiplas comorbidades associadas que podem agravar as taxas de atraso.

\section{REFERÊNCIAS}

1. BAYLEY N. Bayley- escalas de desenvolvimento do bebê e da criança pequena: manual técnico. 3 ed. São Paulo: Person Clinical Brasil, 2018; 165p.

2. BALTIERI L, et al. Desempenho motor de lactentes frequentadores de berçários em creches públicas. Rev Paul Pediatr, 2010; 28(3): 283-9.

3. CARDOSO FGC, et al. Validade concorrente da escala brunet-lézine com a escala bayley para avaliação do desenvolvimento de bebês pré-termo até dois anos. Revista Paulista de Pediatria, 2017; 35(2): 144-150.

4. CERULLO A, et al. Colpocephaly in two siblings: further evidence of a genetic transmission. Developmental Medicine \& Child Neurology, 2000; 42: 280-282.

5. COPPEDE AC, et al. Desempenho motor fino e funcionalidade em crianças com síndrome de Down. Fisioter Pesq, 2012; 19(4): 363-368.

6. FERNANDES LV, et al. Avaliação do neurodesenvolvimento de prematuros de muito baixo peso ao nascer entre 18 e 24 meses de idade corrigida pelas escalas Bayley III. Jornal de Pediatria, 2012; 88(6).

7. JACKSON BJ, et al. Bayley scales of infant development screening test-gross motor subtest: efficacy in determining need for services. Pediatric physical therapy, 2012; 24(1): 58-62.

8. MELO WA, et al. Anomalias congênitas: fatores associados à idade materna em município sul brasileiro, 2000 a 2007 . Rev. Eletr. Enf. [Internet], 2010; 12(1): 73-82.

9. PATNAIK A, et al. A rare case of colpocephaly with macrocephaly successfully treated with ventriculo-peritoneal shunting. JPediatr Neurosci, 2012; 7(2): 150-1.

10. PETRIBU NCL, et al. Achados frequentes na tomografia computadorizada do crânio em neonatos com síndrome congênita pelo vírus Zika confirmada. Radiol Bras, 2018; 51(6): 366-371.

11. RODRIGUES OMPR. Escalas de desenvolvimento infantil e o uso com bebês. Educar em Revista, 2012; 43: 81-100.

12. PUVABANDITSIN S, et al. Colpocephaly: a case report. Am J Perinatol, 2006; 23(5): 295-7.

13. SALDANHA RP, et al. Colpocefalia em recém-nascido: relato de caso e revisão de literatura. Residência Pediátrica, 2017; 7(3): 110-113.

14. SANTOS MM, et al. Comparação do desempenho motor e cognitivo de crianças frequentadoras de creches públicas e particulares. Braz J Phys Ther, 2013; 17(6): 579-587.

15. SARNAT HB, FLORES-SARNAT, L. Molecular genetic and morphologic integration in malformations of the nervous system for etiologic classification. Semin Pediatr Neurol, 2002; 9: 335-44.

16. SOUZA CT, et al. Avaliação do desempenho motor global e em habilidades motoras axiais e apendiculares de lactentes frequentadores de creche. Rev Bras Fisioter, 2010; 14(4): 309-15.

17. ULUDAĞ S, et al. A Very Rare Case of Colpocephaly Associated With Trisomy 18. Gynecol Obstet Reprod Med, 2012; 18: 83-5. 